\title{
EFFECTS OF POSTERIOR CAPSULE STRETCH ON ADHESIVE CAPSULITIS.
}

1. DPT, M.Phil.

Physical Therapist CDA Hospital Islamabad.

2. DPT, M.Phil, PhD Scholar Assistant Professor/ Principal Riphah College of Rehabilitation Sciences, Faisalabad.

3. DPT, M.Phil, PhD Scholar

Associate Professor/HOD Riphah College of Rehabilitation Sciences, Islamabad.

4. MBBS, M.Phil, Ph.D Scholar Associate Professor Department of Physiology. Independent Medical College Faisalabad.

5. DPT, M.Phil Physical Therapist

ISI Special Education School, Hamza Camp Rawalpindi.

6. MBBS, M.Phil, PhD Scholar Assistant Professor Department of Biochemistry Independent Medical College Faisalabad.

Correspondence Address: Dr. Razia Rizwan

Department of Physiology Independent Medical College. Faisalabad.

drrizi92@gmail.com

Article received on: 27/07/2018

Accepted for publication: 05/01/2019

Received after proof reading: 31/07/2019

\section{INTRODUCTION}

The causes of functional limitation among patients suffering from Adhesive Capsulitis are pain and limited range of motion. About $50-80 \%$ suffering from shoulder pain neglect to seek medical attention. Regardless of this, shoulder pain is the third most normal musculoskeletal explanation behind individuals to visit their GPs, and around $15 \%$ of these individuals are referred for physiotherapy in the three years following their underlying interviews. ${ }^{1}$ Walker-Bone et al completed study on extensive scale in 2004 and found that pervasiveness of adhesive capsulitis among females was $10.1 \%$ while for male it was $8.2 \%$. According to study conducted by Bunker in 2009 adhesive capsulitis affects only $0.75 \%$ population. ${ }^{2,3}$

\author{
Madiha Maryam¹, Muhammad Kashif ${ }^{2}$, Abdul Ghafoor Sajjad ${ }^{3}$, Razia Rizwan ${ }^{4}$, Sehrish Ali ${ }^{5}$, \\ Rizwan Ahmad
} Setting: Department of Physiotherapy, Capital Development Authority Hospital Islamabad. Period: October 2015 to March 2016. Materials and Methods: Forty female patients having aged between 40 to 70 years with freezing and frozen stage of adhesive capsulitis were screened excluded from the study. Subjects were randomly place along with conventional manual therapy and electrotherapy whereas the control group had 21 subjects and they were given manual therapy and electrotherapy alone. The Numeric Pain Rating Scale, Shoulder Pain and Disability Index, and Range of Motion of shoulder joint were of Group A was 115.00 22.023 and of the Group B was $81.74 \pm 20.653$ with $p$ value was $P<05$. showed non-significant difference. Conclusion: It is concluded that posterior capsular stretch

Key words: $\quad$ Adhesive Capsulitis, Analgesia, Frozen Shoulders, Mobilization, Posterior Capsular Stretch. posterior capsule stretch on adhesive capsulitis. Professional Med J 2019; 26(8):1272-1277. DOI: 10.29309/TPMJ/2019.26.08.3868

Capsular tightness at the shoulder is generally called as frozen shoulder or adhesive capsulitis. The pervasiveness of the ailment is more in females than males, moderately aged and older more than young population. Some studies indicated the change in pattern of scapulohumeral rhythm as one of the cause of frozen shoulder of unknown etiology particularly in thoracic kyphosis. This is reliable with the fact that women are more affected because they are more prone to developing thoracic kyphosis than men. ${ }^{4}$

To provide the firm basis, a study related to pathology of adhesive capsulitis was done. The patients were first treated by manipulation under anaesthesia and later arthroscopic release of the coracohumeral ligament was performed. 
Biopsies from the site were taken to perform the histological and immunocytochemical analysis and to identify the types of cell present. In conclusion, arthroscopic biopsy material in patients with adhesive capsulitis suggests immunocytochemical evidence of both chronic inflammation and proliferative fibrosis. Together with the presence of high blood supply and nerve tissue, this inflammation and fibrosis explains why adhesive capsulitis is such a painful and stiff condition. ${ }^{5}$ Presence of very high inflammatory cytokines may be associated with the pathogenesis of inflammation leading to fibrosis, which is the characteristic feature of adhesive capsulitis. ${ }^{6}$

Boyle-Walker et al describes in 1997 that over $90 \%$ patients feel pain before stiffness, in the next stage patients reported increased intensity of pain and associated limited range of motion due to stiffness. In this stage pain is the major complaint that affects activities of daily living and also sleep. Some patients also report radiating pain in the arm. (Cyriax1982). Sleep disturbance in the patients is main purpose of consultation. Another study on 223 individuals reported that pain is the only symptom in about $38 \%$ of individuals after a follow up of four years and in $3 \%$ of individuals reported pain and associated functional disability as the major symptoms in such patients. ${ }^{7}$

Multiple studies have looked at the efficacy of rehabilitation following adhesive capsulitis. In general, most of these studies demonstrate various degrees of improvement in pain scores, ROM, and function following various treatment modes. Physiotherapy is becoming a popular nonsurgical treatment especially in the frozen and thawing stage. Some studies have shown that low grade physiotherapy may show better long term outcome as compared to high intensity. ${ }^{8}$ The mobilization techniques plays important role in restoration of biomechanical movements among patients with adhesive capsulitis. ${ }^{9} \mathrm{~A}$ comparative study ${ }^{10}$ has been conducted on two groups. One group was treated with range of motion exercises along with glides and other was treated with active range of motion exercises alone. The group treated with range of motion exercises along with glides showed greater improvement in symptoms of pain and decreased functional ability as compared to the other group. ${ }^{11}$ Author $^{12}$ proposed a study comparing the effectiveness of mid-range mobilization, end-range mobilization and mobilization with movement in treating the stiffness phase of adhesive capsulitis. They stated that end-range mobilization and mobilization with movement were more effective than mid-range mobilization in improving mobility and functional abilities.

Another study reported the effects of end-range mobilization in individuals with decreased range of motion at shoulder joint due to adhesive capsulitis. The study ${ }^{13}$ assess the reliability of techniques and methods used for measuring symptoms associated with adhesive capsulitis. The study also assess the effects of gliding techniques applied at end-range in improving range of motion at shoulder joint by measuring range of motion before and after the application of gliding techniques. ${ }^{14} \mathrm{It}$ supports the theory that end range mobilization is effective in decreasing the risk of progressive stiffness and joint contracture in subjects with frozen shoulder.

\section{MATERIAL \& METHODS}

A randomized controlled study was carried out at Department of Physiotherapy, Capital Development Authority Hospital Islamabad from October 2015 to March 2016. Forty female patients having aged between 40 to 70 years with freezing and frozen stage of adhesive capsulitis were screened out. Adhesive capsulitis secondary to cervical Spondylosis, osteoporosis, direct trauma and any inflammation were excluded from the study. Using non probability purposive sampling technique subjects were divided into two groups on random allocatoin; the experimental group having 19 subjects and control group had 21 subjects. Written consent was taken from every one of the subjects who get enrolled in the study. The Numeric Pain Rating Scale, Shoulder Pain and Disability Index, and Range of Motion of shoulder joint were used as outcome measures. All the patients were assessed at baseline before intervention and at the completion of 6 weeks of treatment. Experimental group received posterior 
capsular stretch (Actively 3 sets of 10 repetitions; passively 3 sets of 10 repetitions) along with conventional manual therapy (Anterior, Posterior and Inferior Glide 3 sets of 10 repetitions each) and electrotherapy (IFC Frequency 4100/4100 for 10 minutes on shoulder with shortwave diathermy 10 minutes). Whereas the control group were given manual therapy (Anterior, Posterior and Inferior Glide 3 sets of 10 repetitions each) and electrotherapy (IFC Frequency $4100 / 4100$ for 10 minutes on shoulder with shortwave diathermy 10 minutes) alone. Data was analyzed $(n=40)$ at baseline and later after 6 weeks of intervention using SPSS version 20. Paired and Independent t-test was applied on Numeric Pain Rating Scale (NPRS), Shoulder Pain and Disability Index (SPADI), and Range of Motion of shoulder joint
(ROM).

\section{RESULTS}

The mean age for the participants was $52.30 \pm 7.95$, the mean age for experimental group was $53.86 \% \pm 8.604$ and that for control group was $50.05 \% \pm 6.827$. The majority $(37.5 \%)$ had onset of pain 3 months before.67.5\% participants had aggravating factor lying on affected side. The relieving factor for majority of patients was NSAIDS intake. $97.5 \%$ patients had intermittent duration of pain.

Given below are the tables for within the group and between the group comparison in experimental (Group A) and control group (Group B).

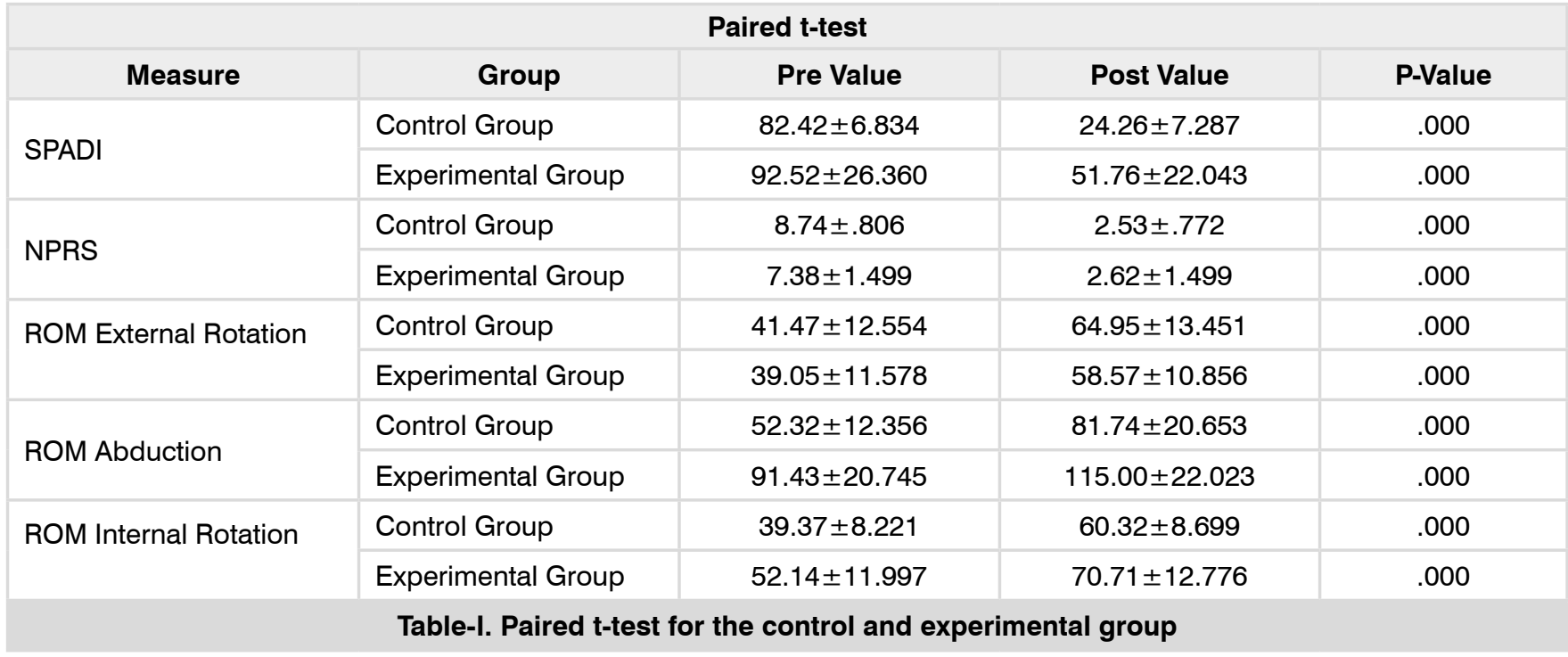

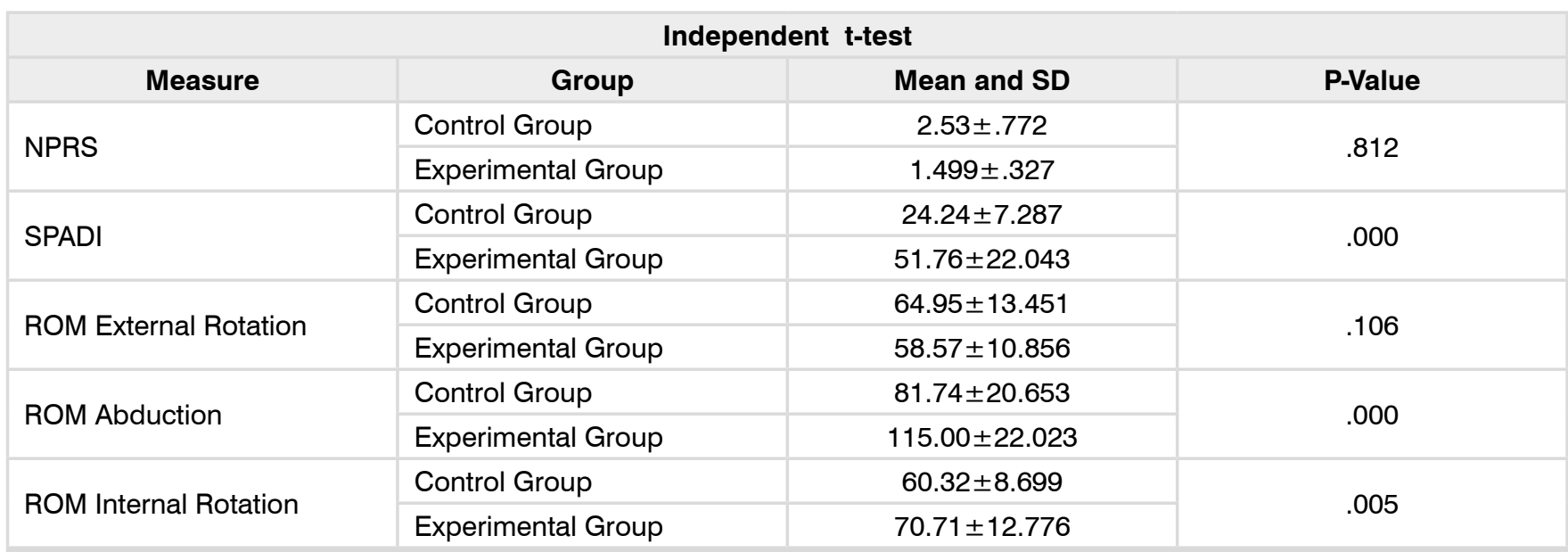

Table-II. Independent t-test between the control and experimental group 


\section{DISCUSSION}

The result of this study show posterior capsular stretch with Kaltenborn mobilization in adhesive capsulitis patients had significant result. The results of study also show significant difference in improving range of motion and quality of life in both control and experimental group as well.

A comparative study conducted by Henricus $M$ Vermeulen et al. in 2006 on the effects of high intensity glides and low intensity glides in patients with frozen shoulder. This study also shows mobilization to be very effective technique in the management of adhesive capsulitis in both groups. ${ }^{15}$

Another study of Henricus M Vermeulen et al. in 2000 was done to assess the role of glides at the in adhesive capsulitis patients. The results showed that intensive mobilization played significant role in management of pain and limited range of motion in patients with frozen shoulder. ${ }^{16}$ In this study we also did grade 3 kaltenborn mobilizations in both groups and came to be very effective in improving range of motion and activities of daily living.

Another review on adhesive capsulitis: diagnosis and therapy was conducted about phases of adhesive capsulitis (freezing, frozen and thawing) and their recommended treatment concluded that pain medications and steroid injections must be used in freezing phase and manual therapy along with exercise in frozen and thawing phases. In resistant cases which show no improvement other surgical treatments was recommended. ${ }^{17}$ the review recommended treatment was mobilization in the second and third phases of adhesive capsulitis. In current study patients in frozen and thawing phases were allocated into two groups, were given two different manual mobilization therapies and significant improvement was seen.

Johnson AJ GJ et al conducted Randomized control trial to compare the effectiveness of posterior and anterior glide mobilization for External rotation range of motion in adhesive capsulitis patients, 20 patients were enrolled and not given any home exercise plan, both glider were given as sustained end range glides.
External rotation range of motion in maximum abduction and pain VAS were used as outcome measures, study concluded that posterior glide was more effective in improving external rotation range of motion in full abduction than anterior glide. $^{18}$

One of the study done by Jing-lan Yang et al. in 2007 was carried out to compare the use of 3 mobilization techniques - end-range mobilization (ERM), mid-range mobilization (MRM), and mobilization with movement (MWM) -in the management of subjects with frozen shoulder syndrome (FSS).this study concluded that ERM and MWM were more effective than MRM in increasing mobility and functional ability. ${ }^{19}$ My study results also support this study.

Syed Shakil-ur-Rehman et al conducted an RCT comparison between kalternborn technique and scapular mobility technique in patients of adhesive capsulitis with abduction range of motion above $90^{\circ}$ was done. Forty seven patients were randomly allocated in two groups. One group was treated with sustained stretch mobilization and other was treated with scapular mobilization and measuring tool was abduction ROM. Result of study showed that sustained stretch mobilization was more effective than scapular mobilization. in our study posterior capsular stretch is applied along with mobilization which causes significant improvement in increasing range of motion and functional disability.

Another study done by Andrea J. Johnson, DPTSc was the Effect of Anterior Versus Posterior Glide Joint Mobilization on External Rotation Range of Motion in Patients with Shoulder Adhesive Capsulitis. ${ }^{20}$ The results showed that posteriorly directed joint mobilization techniques were more effective than anteriorly directed joint mobilization techniques. In our study we also applied anterior, posterior and inferior glides and results shows that they increases range of motion and activities of daily living.

A study done by Antony Paul affiliated with Department of PMR, St John's Medical College \& Hospital, Joshua Samuel Rajkumar in 2014 
was carried out to find effectiveness of sustained stretching of inferior capsule in the management of frozen shoulder. ${ }^{21}$ They concluded that stretching along with physiotherapy improved shoulder function compared with physiotherapyalone. In our study we applied posterior capsular stretch along with mobilization in experimental group. The results of our study support the literature review and shows significant improvement in range of motion and decrease in shoulder disability.it shows that posterior capsular stretch along with mobilization plays very important role in the management of adhesive capsulitis.

There was a RCT study done by ${ }^{21}$ in which they compare stretching procedures for posterior shoulder tightness. The results of the study showed that stretching procedures improved internal range of motion than no stretching. Our study also support this literature review as posterior capsular stretching along with kaltenborn mobilization increases range and functional ability as compare to mobilization alone.

\section{CONCLUSION}

Current study showed marked improvement in experimental group as compared to control group. Posterior capsular stretch with mobilization can bring better results in individuals with Adhesive capsulitis. This study demonstrated significant difference in improving range of motion, functional disability and quality of life between both the groups.

Copyright@ 05 Jan, 2019.

\section{REFERENCES}

1. Linsell L, Dawson J, Zondervan K, Rose P, Randall T, Fitzpatrick $R$, et al. Prevalence and incidence of adults consulting for shoulder conditions in UK primary care; Patterns of diagnosis and referral. Rheumatology. 2005;45(2):215-21.

2. Bunker T. Time for a new name for frozen shouldercontracture of the shoulder. Shoulder \& Elbow. 2009;1(1):4-9.

3. Walker Bone K, Palmer KT, Reading I, Coggon D, Cooper C. Prevalence and impact of musculoskeletal disorders of the upper limb in the general population. Arthritis care \& research. 2004;51(4):642-51.
4. Warth RJ, Millett PJ. Physical Examination of the Shoulder: Springer; 2015.

5. Hawker GA, Mian S, Kendzerska T, French M. Measures of adult pain: Visual analog scale for pain (vas pain), numeric rating scale for pain (nrs pain), mcgill pain questionnaire (mpq), short-form mcgill pain questionnaire (sf-mpq), chronic pain grade scale (cpgs), short form-36 bodily pain scale (sf36 bps), and measure of intermittent and constant osteoarthritis pain (icoap). Arthritis care \& research. 2011 Nov;63(S11):S240-52.

6. Van der Windt D, Koes BW, de Jong BA, Bouter LM. Shoulder disorders in general practice: Incidence, patient characteristics, and management. Annals of the rheumatic diseases. 1995;54(12):959-64.

7. Hanchard NC, Goodchild L, Thompson J, O'Brien T, Davison D, Richardson C. Evidence-based clinical guidelines for the diagnosis, assessment and physiotherapy management of contracted (frozen) shoulder: Quick reference summary. Physiotherapy. 2012;98(2):117-20.

8. Hoy D, Bain C, Williams G, March L, Brooks P, Blyth F, et al. A systematic review of the global prevalence of low back pain. Arthritis \& Rheumatism. 2012;64(6):2028-37.

9. Donatelli R, Greenfield B. Case study: rehabilitation of a stiff and painful shoulder: a biomechanical approach. Journal of Orthopaedic \& Sports Physical Therapy. 1987 Sep;9(3):118-26.

10. Nicholson GG. The effects of passive joint mobilization on pain and hypomobility associated with adhesive capsulitis of the shoulder. Journal of Orthopaedic \& Sports Physical Therapy. 1985 Feb;6(4):238-46.

11. Walmsley S, Rivett DA, Osmotherly PG. Adhesive capsulitis: establishing consensus on clinical identifiers for stage 1 using the DELPHI technique. Physical therapy. 2009 Sep 1;89(9):906-17.

12. Resort HRHC, San Antonio S, Course TP. The logo for The Society for Pediatric Radiology was designed by Tamar Kahane Oestreich of Cincinnati, Ohio in 1985. Pediatr Radiol. 2013;43(2):S205-S458.

13. Lugger B. EU Project GAMBA: Compendiun to the manual-basics, need-to-know history. Bioceram Dev Appl. 2013;3(069):2.

14. Marras WS, Cutlip RG, Burt SE, Waters TR. National occupational research agenda (NORA) future directions in occupational musculoskeletal disorder health research. Applied ergonomics. 2009;40(1):1522. 
15. Vermeulen HM, Rozing PM, Obermann WR, Le Cessie $\mathrm{S}$, Vlieland TPV. Comparison of high-grade and lowgrade mobilization techniques in the management of adhesive capsulitis of the shoulder: Randomized controlled trial. Physical therapy. 2006;86(3):355-68.

16. Vermeulen HM, Obermann WR, Burger BJ, Kok GJ, Rozing PM, van den Ende $\mathrm{CH}$. End-range mobilization techniques in adhesive capsulitis of the shoulder joint: a multiple-subject case report. Physical Therapy. 2000 Dec 1;80(12):1204-13.

17. Schultheis A, Reichwein F, Nebelung W. Frozen shoulder. Diagnosis and therapy. Der Orthopade. 2008 Nov;37(11):1065-6.

18. Johnson AJ, Godges JJ, Zimmerman GJ, Ounanian LL. The effect of anterior versus posterior glide joint mobilization on external rotation range of motion in patients with shoulder adhesive capsulitis. journal of orthopaedic \& sports physical therapy. 2007 Mar;37(3):88-99.
19. Yang J-I, Chang C-w, Chen S-y, Wang S-F, Lin J-j. Mobilization techniques in subjects with frozen shoulder syndrome: randomized multiple-treatment trial. Physical therapy. 2007;87(10):1307-15.

20. Johnson AJ, Godges JJ, Zimmerman GJ, Ounanian LL. The effect of anterior versus posterior glide joint mobilization on external rotation range of motion in patients with shoulder adhesive capsulitis. journal of orthopaedic \& sports physical therapy. 2007;37(3):8899.

21. Paul A, Rajkumar JS, Peter S, Lambert L. Effectiveness of sustained stretching of the inferior capsule in the management of a frozen shoulder. Clinical Orthopaedics and Related Research ${ }^{\circledR}$. 2014;472(7):2262-8.

\begin{tabular}{|c|c|c|c|}
\hline \multicolumn{4}{|c|}{ AUTHORSHIP AND CONTRIBUTION DECLARATION } \\
\hline Sr. \# & Author-s Full Name & Contribution to the paper & Author $=\mathbf{s}$ Signature \\
\hline 1 & Madiha Maryam & Conceive idea, study design. & \\
\hline 2 & Muhammad Kashif & $\begin{array}{l}\text { Data collection \& Manuscript } \\
\text { writing. }\end{array}$ & \\
\hline 3 & Abdul Ghafoor Sajjad & Compiling the results. & \\
\hline 4 & Razia Rizwan & Literature review. & \\
\hline 5 & Sehrish Ali & Interduction and references. & \\
\hline 6 & Rizwan Ahmad & Final proof reading of article. & \\
\hline
\end{tabular}

